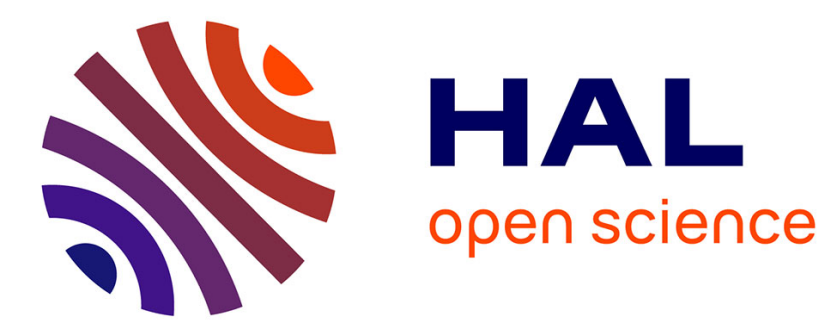

\title{
Relationship between spike worm (Raghuva albipunctella) infestation and flowering of pearl millet, and some sources of resistance \\ Ruparao Tulashiramji Gahukar
}

\section{- To cite this version:}

Ruparao Tulashiramji Gahukar. Relationship between spike worm (Raghuva albipunctella) infestation and flowering of pearl millet, and some sources of resistance. Agronomie, 1987, 7 (8), pp.595-598. hal00885031

\section{HAL Id: hal-00885031 \\ https://hal.science/hal-00885031}

Submitted on 1 Jan 1987

HAL is a multi-disciplinary open access archive for the deposit and dissemination of scientific research documents, whether they are published or not. The documents may come from teaching and research institutions in France or abroad, or from public or private research centers.
L'archive ouverte pluridisciplinaire HAL, est destinée au dépôt et à la diffusion de documents scientifiques de niveau recherche, publiés ou non, émanant des établissements d'enseignement et de recherche français ou étrangers, des laboratoires publics ou privés. 


\title{
Relationship between spike worm (Raghuva albipunctella) infestation and flowering of pearl millet, and some sources of resistance
}

\author{
Ruparao Tulashiramji GAHUKAR (*) \\ International Crops Research Institute for the Semi-Arid Tropics (ICRISAT), B.P. 51, Bambey, Senegal
}

Spike worms have recently been reported as a potential threat to millet production in the Sub-Sahelian zone of West Africa. Infestation of spike worm (Raghuva albipunctella de Joannis) in indigenous and exotic millet genotypes was studied during 1979-81 at Bambey, Senegal. The earliness or lateness of flowering is an important factor in host plant exposure to pest attack and subsequent pest damage. Hybrid ICH-165, synthetics ICMS7838, ICMS-7703 and traditional varieties Souna and CIVT were found resistant to pest attack.

Additional key words : Sahel, flowering period, resistant genotypes.

Mots clés additionnels : Sahel, période de floraison, génotypes résistants.

\section{INTRODUCTION}

Pearl millet (Pennisetum americanum L.) is a major cereal food crop in Senegal. Among about 100 insect pests recorded on the millet crop (RISBEC, 1950 ; NDOYE, 1979a), certain lepidoptera infest the spikes during flowering and grain maturation. Among these, since the drought of 1972-74, larvae of the genera Masalia Moore and Raghuva Moore (Noctuidae) have been reported as a potential threat to millet production in the subsahelian zone of West Africa (VERCAMBRE, 1978).

Several species of Masalia have been identified from Africa and 7 species are present in Senegal (SEYMOUR, 1973 ; VERCAMBRE, 1979). During the last few years using light-trap and pest-rearing studies, we have identified species of Raghuva and Adisura callina

$\left({ }^{*}\right)$ Present address : Plot 220, Reshimbag, Nagpur 44009, India.
Baker (LAPORTE, 1977 ; NDOYE, 1979c). Raghuva albipunctella De Joannis was the most abundant comprising $83.5 \%$ of the moths captured in light traps and $95 \%$ of the larval population observed on millet spikes (NDOYE, 1979a, $b$ ). This pest is distributed throughout millet growing-areas in the northern and south-central parts of Senegal.

Crop damage is caused by larval feeding on millet heads. Larvae of the first two instars feed on the flowers leaving behind empty glumes. Older larvae cut the floral peduncles and make their way between rachis and flowers, thereby pushing out the destroyed flowers. This results in the characteristic spiral shaped damage on the spike. As many as 51 larvae may infest a single spike and the pest may cause up to $50 \%$ loss in grain yield (VERCAMBRE, 1978).

As control measures, deep ploughing at the end of crop season to check the population of diapausing pupae in the soil (VERCAMBre, 1978), and the 
treatment of flowering spikes with endosulfan or chlorodimefon to kill young larvae (ANONYMOUS, 1977) have been recommended. It is realised, however, that in present peasant agriculture, pesticide applications may not be practical and economical. GAHUKAR (1981) and GUEREMONT (1982) have reported a few larval parasites from sahelian countries. However, their effectiveness in reducing crop damage and controlling pest populations is doubtful as they appear after the majority of larvae have penetrated into the soil for pupation.

While varietal resistance is an integral part of pest management strategy, little information is currently available. Thus, we studied the effect of flowering period on pest incidence and evaluated some local and introduced millet genotypes for their performance under natural pest infestation.

\section{MATERIALS AND METHODS}

Seven millet trials (table 1) of the millet breeding program of ICRISAT were evaluated at National Agricultural Research Center in Bambey. Date of sowing varied annually depending upon the onset of rains : 19 June 1979, 7 August 1980 and 7 July 1981.

The genetic pool consisted of traditional varieties from Senegal (Souna), Niger (CIVT-II) and Nigeria (Ex-Bornu) and introduced entries from ICRISAT program in India such as hybrids, (ICH-165, ICH-220, NBH-3), experimental varieties (WC-C-75), progeny variety (IVS-5454) and synthetics (all ICMS entries). A hybrid NHB-3 served as a check.

Plots of $18-20 \mathrm{~m}^{2}$ for each entry were arranged in a completely randomized block design with 3-5 replications in rows $80 \mathrm{~cm}$ apart. NPK fertilizer 10.21.21 was applied at $150 \mathrm{~kg} / \mathrm{ha}$ before sowing. Urea was applied twice at $50 \mathrm{~kg} / \mathrm{ha}$ : at thinning (10-15 days after plant emergence) and at boot stage (40-50 days after first application).

Observations on pest damage were taken in each plot 2 weeks after $50 \%$ flowering on 200 randomly selected spikes in the three central rows. Larval counts were made on 25 spikes. Grain yield per plot was recorded.

Data collected during the three years were statistically analysed, and simple correlations established.

\section{RESULTS AND DISCUSSION}

\section{A. Pest incidence and its relation with millet flowering}

The pest incidence varied considerably among years and within a year in different trials. Although larvae of both Masalia and Raghuva were present on spikes, only Raghuva larvae were recorded as they represented more than $85 \%$ of the total population. Furthermore, it was observed that $R$. albipunctella was the predominant species. Correlations between pest incidence, larval numbers and grain yield were not consistent during years and therefore are not dealt with here.

1979 : The percentage of infested spikes ranged from 35 to 100 in 5 trials (table 1). In general, early flowering entries received higher pest attack but these correlations were significant $(P=0.01)$ only for IPMAT and WART. Mean larval number on 25 spikes varied from 5 to 34 and hybrids harboured larger population.

1980 : In the 1980 crop season, pest incidence was comparatively low. The range of percent infested spikes recorded in 6 trials was 23-72 (table 1). Entries in SRT flowered lately ( 52 days) and had less pest attack. The relationship between flowering time and pest infestation was significant only for PMST entries ( $p=0.01$, $r=0.57)$. Larval numbers were not always associated with flowering.

TABLEAU 1

Incidence of $\mathrm{R}$. albipunctella and period to $50 \%$ flowering in pearl millet trials and their correlations, Bambey, $1979-81$. Incidence de R. albipunctella et période de 50 p. 100 floraison dans les essais de petit mil et leurs corrélations, Bambey, 1979-81.

\begin{tabular}{|c|c|c|c|c|c|c|c|c|c|c|c|c|c|}
\hline \multirow[t]{2}{*}{ Year } & \multirow[t]{2}{*}{ Trial } & \multicolumn{3}{|c|}{$\begin{array}{l}\text { Spikes attacked } \\
\text { in per cent }\end{array}$} & \multicolumn{3}{|c|}{$\begin{array}{c}\text { Number of } \\
\text { larvae } / 25 \text { spikes }\end{array}$} & \multicolumn{3}{|c|}{$\begin{array}{l}\text { Days to } \\
50 \% \text { flowering } \\
\end{array}$} & \multirow[t]{2}{*}{ DF } & \multicolumn{2}{|c|}{$\begin{array}{l}\text { Correlations of lateness } \\
\text { of flowering period with }\end{array}$} \\
\hline & & range & mean & $\begin{array}{l}\text { SEm } \\
( \pm)\end{array}$ & range & mean & $\begin{array}{l}\text { SEm } \\
( \pm)\end{array}$ & range & mean & $\begin{array}{l}\text { SEm } \\
( \pm)\end{array}$ & & $\begin{array}{l}\text { spike } \\
\text { attack }\end{array}$ & $\begin{array}{c}\text { larval } \\
\text { number }\end{array}$ \\
\hline \multirow[t]{5}{*}{1979} & IPMAT & $45-99$ & 92.7 & 9.8 & $2-12$ & 7.3 & 5.2 & $41-57$ & 46.0 & 5.4 & 22 & $-0.58 * *$ & +0.17 \\
\hline & PMST & $63-99$ & 93.8 & 10.1 & $2-13$ & 6.8 & 3.1 & 43-64 & 47.1 & 1.2 & 22 & -0.40 & -0.12 \\
\hline & PMHT & $62-99$ & 93.2 & 9.5 & $12-55$ & 33.6 & 9.9 & $44-62$ & 47.8 & 1.3 & 26 & -0.12 & -0.35 \\
\hline & WART & $35-98$ & 67.5 & 13.8 & $10-28$ & 20.9 & 5.7 & $47-63$ & 56.4 & 3.2 & 10 & $-0.92^{* *}$ & $-0.80^{* *}$ \\
\hline & EVT & $65-100$ & 93.9 & 10.1 & $1-12$ & 5.5 & 3.5 & $43-49$ & 46.9 & 4.2 & 26 & -0.11 & +0.09 \\
\hline \multirow[t]{6}{*}{1980} & IPMAT & $32-57$ & 44.1 & 5.8 & $1-6$ & 4.1 & 1.8 & $42-47$ & 44.4 & 1.5 & 19 & -0.19 & +0.13 \\
\hline & PMST & $23-51$ & 37.6 & 6.6 & $1-2$ & 1.0 & 0.6 & $43-47$ & 44.9 & 1.1 & 18 & $-0.57^{* *}$ & +0.27 \\
\hline & WART & $29-50$ & 38.8 & 6.4 & $1-3$ & 0.8 & 0.9 & $46-58$ & 50.1 & 1.9 & 23 & -0.26 & -0.39 \\
\hline & EVT & $36-72$ & 50.6 & 4.3 & $1-3$ & 1.0 & 0.7 & $42-47$ & 45.1 & 0.9 & 30 & +0.02 & -0.12 \\
\hline & ELVT & $44-68$ & 56.6 & 6.1 & $1-6$ & 3.3 & 1.4 & $42-46$ & 44.7 & 1.0 & 30 & -0.33 & +0.12 \\
\hline & SRT & $26-45$ & 33.5 & 6.2 & $1-2$ & 1.0 & 0.6 & $43-58$ & 52.4 & 1.9 & 14 & +0.18 & -0.17 \\
\hline \multirow[t]{4}{*}{1981} & IPMAT & $9-26$ & 15.8 & 5.0 & $5-14$ & 10.1 & 3.5 & $48-57$ & 51.6 & 1.5 & 19 & $-0.45^{*}$ & +0.0 \\
\hline & PMST & $1-15$ & 5.4 & 5.8 & $1-13$ & 5.8 & 4.7 & $39-48$ & 39.2 & 7.4 & 23 & -0.21 & -0.35 \\
\hline & PMHT & $15-42$ & 21.6 & 6.4 & $13-23$ & 14.6 & 3.7 & $53-57$ & 53.8 & 2.7 & 4 & $-0.91 *$ & $-0.85^{*}$ \\
\hline & ELVT & $2-14$ & 11.9 & 6.9 & $2-43$ & 10.3 & 11.8 & $51-59$ & 55.9 & 1.9 & 18 & $-0.86^{* *}$ & -0.43 \\
\hline
\end{tabular}


1981: All trials suffered from drought during flowering and entries in synthetics and hybrids suffered the most. Percentage of infested heads was low irrespective of larval population. Of 4 trials, hybrids (PMHT) had the highest infestation rate $(22 \%)$ and larval population (15/25 spikes) (table 1). Synthetics (PMST) flowered as early as 39 days and escaped worm attack. Significant correlations were present at $\mathrm{p}=0.01$ between flowering time and pest infestation in IPMAT and PMST and at $p=0.05$ in ELVT.

\section{B. Reaction of some genotypes to pest attack}

1979 : Among 17 promising genotypes, only Souna and CIVT-II had less than $45 \%$ spikes attacked and a maximum of $97 \%$ was found on IVS-5454 (table 2). However, CIVT-II harboured comparatively more larvae. Higher yields were obtained from Souna followed by ICMS-7838, ICMS-7816, ICMS-7819 and ICH-165. It seems that ICMS-7816 and ICH-165 could tolerate larval feeding without their grain filling being affected.

\section{TABLEAU 2}

Incidence of $\mathrm{R}$. albipunctella, period to $50 \%$ flowering and grain yield in 17 selected genotypes of pearl millet, Bambey, 1979. Incidence de $\mathbf{R}$. albipunctella, période de $50 \mathrm{p}$. 100 floraison et rendement en graines dans 17 génotypes sélectionnes de petit mil, Bambey, 1979.

\begin{tabular}{|c|c|c|c|c|}
\hline Genotype & $\begin{array}{l}\% \text { spike } \\
\text { attacked }\end{array}$ & $\begin{array}{l}\text { Number of } \\
\text { larvae on } \\
25 \text { spikes }\end{array}$ & $\begin{array}{c}\text { Grain } \\
\text { yield } \\
(\mathrm{kg} / \mathrm{ha})\end{array}$ & $\begin{array}{c}\text { Days to } \\
50 \% \\
\text { flowering }\end{array}$ \\
\hline Souna & 44.7 & 3.7 & 2993 & 56 \\
\hline ICMS-7818 & 85.9 & 10.3 & 1350 & 44 \\
\hline $\mathrm{ICH}-165$ & 94.9 & 12.0 & 2173 & 50 \\
\hline ICMS-7703 & 96.9 & 11.0 & 680 & 42 \\
\hline ICM-220 & 99.0 & 3.0 & 406 & 41 \\
\hline WC-C-75 & 95.3 & 11.7 & 880 & 43 \\
\hline IVS-5454 & 97.1 & 8.7 & 1240 & 45 \\
\hline CIVT-II & 35.2 & 2.7 & 1533 & 61 \\
\hline EX BORNU & 72.1 & 23.7 & 1280 & 48 \\
\hline ICMS-7838 & 63.4 & 4.0 & 2376 & 53 \\
\hline ICMS-7857 & 95.8 & 2.3 & 2140 & 46 \\
\hline ICMS-7806 & 96.7 & 6.7 & 1753 & 47 \\
\hline ICMS-7825 & 96.1 & 7.0 & 2005 & 49 \\
\hline ICMS-7835 & 90.0 & 2.3 & 1686 & 45 \\
\hline ICMS-7816 & 96.6 & 11.3 & 2213 & 46 \\
\hline ICMS-7819 & 96.8 & 7.3 & 2211 & 47 \\
\hline $\begin{array}{l}\text { NHB-3 } \\
\text { (susceptible check) }\end{array}$ & 95.5 & 12.7 & 613 & 41 \\
\hline Mean & 85.4 & 8.2 & 1619.8 & 47.6 \\
\hline $\mathrm{CV}$ & 23.6 & 61.9 & 27.1 & 9.0 \\
\hline LSD $\quad 0.05$ & 23.8 & 6.0 & 518 & 5 \\
\hline 0.01 & 32.0 & 8.1 & 698 & 7 \\
\hline F Test & HS & HS & HS & HS \\
\hline
\end{tabular}

1980 : There was little variation in percentage spikes attacked (mean $38 \%$ ) among 16 selected entries (table 3). Entries ICMS-7838, ICH-165, CIVT-II, Souna and IVS-5454 were relatively resistant. Yield of more than $2200 \mathrm{~kg} /$ ha was obtained from ICMS-7703, ICH-165 and Souna.

1981: The check entry NHB-3 had the highest spike infestation $(27 \%)$ whereas synthetics ICMS-7914, ICMS-7903 and ICMS-7908 were resistant to worm
TABLEAU 3

Incidence of $\mathrm{R}$. albipunctella, period to $50 \%$ flowering and grain yield in 16 selected genotypes of pearl millet, Bambey, 1980. Incidence de $\mathrm{R}$. albipunctella, période de 50 p. 100 floraison et rendement en graines dans 16 génotypes sélectionnés de petit mil, Bambey, 1980.

\begin{tabular}{|c|c|c|c|c|}
\hline Genotype & $\begin{array}{l}\% \text { spike } \\
\text { attacked }\end{array}$ & $\begin{array}{l}\text { Number of } \\
\text { larvae on } \\
25 \text { spikes }\end{array}$ & $\begin{array}{l}\text { Grain } \\
\text { yield } \\
(\mathrm{kg} / \mathrm{ha})\end{array}$ & $\begin{array}{c}\text { Days to } \\
50 \% \\
\text { flowering }\end{array}$ \\
\hline Souna & 32.0 & 6.0 & 2288 & 43 \\
\hline ICMS-7818 & 40.0 & 4.3 & 1719 & 48 \\
\hline ICH-165 & 34.0 & 4.0 & 2654 & 45 \\
\hline ICMS-7703 & 40.6 & 3.6 & 2385 & 43 \\
\hline ICH-220 & 42.0 & 3.6 & 2108 & 42 \\
\hline WC-C-75 & 52.6 & 3.6 & 1696 & 44 \\
\hline IVS-5454 & 34.6 & 5.6 & 1779 & 45 \\
\hline CIVT-II & 34.7 & 0.3 & 2089 & 50 \\
\hline ICMS-7838 & 24.6 & 1.0 & 2045 & 45 \\
\hline ICMS-7857 & 34.0 & 1.0 & 1718 & 46 \\
\hline ICMS-7825 & 36.6 & 1.0 & 1786 & 45 \\
\hline ICMS-7835 & 44.0 & 1.3 & 1890 & 45 \\
\hline ICMS-7816 & 40.0 & 1.0 & 1831 & 44 \\
\hline ICMS-7819 & 41.3 & 1.6 & 1406 & 45 \\
\hline EX BORNU & 36.0 & 2.3 & 1852 & 51 \\
\hline $\begin{array}{l}\text { NHB-3 } \\
\text { (susceptible check) }\end{array}$ & 41.3 & 3.0 & 584 & 44 \\
\hline Mean & 38.0 & 2.7 & 1864 & 45.6 \\
\hline $\mathrm{CV}$ & 33.3 & 80.7 & 25.4 & 5.3 \\
\hline LSD $\quad 0.05$ & - & - & 560 & 3 \\
\hline 0.01 & - & - & 755 & 4 \\
\hline F Test & NS & NS & HS & HS \\
\hline
\end{tabular}

attack (table 4). ICH-220 yielded $771 \mathrm{~kg} / \mathrm{ha}$ as it flowered early (43 days) and escaped from moth flights and severe drought during flowering.

Many factors may be involved in the millet-worm relationship, such as between floral density and egg laying, between density, length and orientation of awn

TABLEAU 4

Incidence of $\mathrm{R}$. albipunctella, period to $50 \%$ flowering and grain yield in 12 selected genotypes of pearl millet, Bambey, 1981. Incidence de $\mathrm{R}$. albipunctella, période de 50 p. 100 floraison et rendement en graines dans 12 génotypes sélectionnés de petit mil, Bambey, 1981.

\begin{tabular}{lrccc}
\hline \hline Genotype & $\begin{array}{r}\text { \% spike } \\
\text { attacked }\end{array}$ & $\begin{array}{c}\text { Number of } \\
\text { larvae on } \\
\text { 25 spikes }\end{array}$ & $\begin{array}{c}\text { Grain } \\
\text { yield } \\
(\mathrm{kg} / \mathrm{ha})\end{array}$ & $\begin{array}{c}\text { Days to } \\
50 \% \\
\text { flowering }\end{array}$ \\
\hline Souna & 9.5 & 12.0 & 662 & 56 \\
ICH-165 & 17.0 & 8.5 & 633 & 53 \\
ICMS-7703 & 10.5 & 12.5 & 697 & 53 \\
ICH-220 & 18.2 & 13.0 & 771 & 43 \\
WC-C-75 & 17.7 & 9.5 & 632 & 51 \\
ICMS-7914 & 3.2 & 5.0 & 542 & 59 \\
ICMS-7903 & 0.5 & 3.5 & 246 & 60 \\
ICMS-7918 & 7.2 & 12.2 & 598 & 62 \\
ICMS-7908 & 0.7 & 1.5 & 282 & 72 \\
ICMS-7916 & 22.0 & 13.0 & 696 & 64 \\
ICMS-7806 & 6.7 & 12.0 & 566 & 59 \\
NHB-3 & 27.5 & 16.0 & 673 & 49 \\
(susceptible check) & & & & \\
\hline Mean & 11.7 & 9.9 & 583 & 56.9 \\
CV & 48.7 & 64.4 & 22.5 & 13.0 \\
LSD 0.05 & 8.9 & - & 204 & - \\
$\quad 0.01$ & 12.6 & - & $\overline{\mathrm{S}}$ & $-\mathrm{NS}$ \\
F Test & $\mathrm{HS}$ & $\mathrm{NS}$ & $\mathrm{S}$ & \\
\hline \hline
\end{tabular}


and oviposition preference and between spike compactness and antibiosis effect on larval population. Of these, only the first aspect has been studied, by VERCAMBRE (1978) and GUEREMONT (1982) showing a negative relationship. Based on evaluation of 3 years' data of the present study, 3 entries from introduced material (ICMS-7703, ICMS-7838 and ICH-165) and 2 traditional varieties (Souna and CIVT-II) are selected as resistant for their earliness or lateness of flowering that escape exposure to pest attack. Other factors such as mentioned above may also be involved and need further study.

Large scale cultivation of hybrids and synthetics under present farming conditions in Senegal may not be feasible because of lack of seed and fertiliser. Therefore, it may be appropriate to incorporate the suitable characters of resistance and yield potentiality in introduced genotypes into existing local millets.

Recu le 29 octobre 1986. Accepté le 14 avril 1987.

\section{ACKNOWLEDGMENTS}

We wish to thank Dr. A. LAMBERT, former millet breeder of ICRISAT Cooperative Program in Senegal, for his collaboration, Mr M. GALiBa of CNRA, Bambey, for his help and advice in data analysis.

\section{REFERENCES}

Anonymous, 1977. Rapport d'activités, cultures exondées. Division d'Entomologie, Centre National de Recherches Agronomiques, Bambey, Sénégal, $44 \mathrm{p}$.

Gahukar R. T., 1981. Biological control of insect pests of sorghum and pearl millet in West Africa, p. 69-95. Proc. of int. Conf. on biol. control of pests : its potential in West Africa. USAID, Dakar, Senegal, Feb. 9-13, 1981.

Gueremont H., 1982. Rapport annuel de Recherches pour l'année 1981 : Etudes sur l'entomofaune du mil. Centre National de Recherches Agronomiques, Tarna, Maradi, Niger, 57 p.

Laporte B., 1977. Note concernant des Melicleptrinae dont les chenilles sont mineuses des chandelles de mil au Sénégal. Agron. Trop., 32, 429-432.

Ndoye M., 1979a. L'entomofaune nuisible au mil à chandelle (Pennisetum typhoïdes) au Sénégal, p. 515-530. C. R. Congr., sur la lutte contre les insectes en milieu tropical. Chambre de Commerce et d'Industrie, Marseille, France, 13-16 mars 1979.
Ndoye M., 1979b. New millet spike pests in Senegal and the Sahelian zone. FAO Plant Prot. Bull., 27, 7-8.

Ndoye M., 1979c. Rapport d'activités 1977-78. Division d'entomologie, Centre National de Recherches Agronomiques, Bambey, Sénégal, $19 \mathrm{p}$.

Risbec J., 1950. La faune entomologique des cultures au Sénégal et au Soudan Français. Gouvernement Général de l'Afrique Occidentale Française. Jouve, Paris, 638 p.

Seymour P. R., 1973. A revision of the genus Masalia (Lepidoptera, Heliothidinae). Bull. Brit. Mus. (NH), London, $\mathrm{n}^{\circ} 27,100 \mathrm{p}$.

Vercambre B., 1978. Raghuva spp., Masalia sp., chenilles des chandelles du mil en zone sahélienne. Agron. Trop., 33, 62-79.

Vercambre B., 1979. Raghuva spp. et Masalia nubila Hampson, chenilles des chandelles du mil en zone sahélienne, p. 503-514. C. $R$. Congr. sur la lutte contre les insectes en milieu tropical. Chambre de commerce et d'Industrie, Marseille, France, 13-16 mars 1979. 